\title{
Toughness of the corona of two graphs
}

\author{
R.M. Casablanca, A. Diánez and P. García-Vázquez \\ Departamento de Matemática Aplicada I, Universidad de Sevilla, Sevilla, Spain
}

\begin{abstract}
The toughness of a non-complete graph $G=(V, E)$ is defined as $\tau(G)=\min \{|S| / \omega(G-S)\}$, where the minimum is taken over all cutsets $S$ of vertices of $G$ and $\omega(G-S)$ denotes the number of components of the resultant graph $G-S$ by deletion of $S$. The corona of two graphs $G$ and $H$, written as $G \circ H$, is the graph obtained by taking one copy of $G$ and $|V(G)|$ copies of $H$, and then joining the $i$ th vertex of $G$ to every vertex in the $i$ th copy of $H$. In this paper, we investigate the toughness of this kind of graphs and obtain the exact value for the corona of two graphs belonging to some families as paths, cycles, stars, wheels or complete graphs.
\end{abstract}

Keywords: connectivity; toughness; networks; vulnerability

\section{Introduction}

Throughout this paper, all the graphs are simple, that is, without loops and multiple edges. Notations and terminology not explicitly given here can be found in the book by Chartrand and Lesniak [9].

Let $G$ be a graph with vertex set $V(G)$ and edge set $E(G)$. The graph $G$ is called connected if every pair of vertices is joined by a path. A cutset in a graph $G$ is a subset $S \subset V(G)$ of vertices of $G$ such that $G-S$ is not connected. The existence of a cutset is always guaranteed in every graph different from a complete graph $K_{n}$. The index of connectivity of $G$, denoted by $\kappa(G)$, is defined as the minimum cardinality over all cutsets of $G$, if $G$ is a non-complete graph, or $|V(G)|-1$, otherwise.

There are several measures of vulnerability of a network $[6,18,21,22]$. The vulnerability parameters one generally encounters are the indices of connectivity and edge-connectivity. These two parameters give the minimum cost to disrupt the network, but they do not take into account what remains after destruction. To measure the vulnerability of networks more properly, some vulnerability parameters have been introduced and studied. Among them are toughness [3,17,19,20,23], integrity [2], neighbour-integrity [1,26], scattering number [27], tenacity [24] and several variants of connectivity and edge-connectivity called conditional connectivity $[5,7,11,12,16]$, each of 
which measures not only the difficulty of breaking down the network but also the effect of the damage. In general, for most of the aforementioned parameters, the corresponding computing problem is NP-hard. So it is of interest to give the formulae or algorithms for computing these parameters for special classes of graphs. For our purpose, we deal with the notion of toughness, introduced by Chvátal [10], which pays special attention to the relationship between the cardinality of the rupture set in the network and the number of components after rupture. The parameter is defined as

$$
\tau(G)=\min \{|S| / \omega(G-S): S \subseteq J(G)\},
$$

where $J(G)=\{S \subset V(G): S$ is a cutset of $G$ or $G-S$ is an isolated vertex $\}$ and $\omega(G-S)$ denotes the number of components in the resultant graph $G-S$ by removing $S$.

Since this parameter was introduced, a lot of research has been done, mainly relating toughness conditions to the existence of cycle structures. Historically, most of the research was based on a number of conjectures in [10]. Some of most interesting results are addressed in $[4,8,13]$. However, exact values of $\tau(G)$ are known only for a few families of graphs $G$, as paths and cycles [25], the Cartesian product of two complete graphs [10] and of paths and/or cycles [15] and the composition of two graphs, one of them being a path, a cycle or a complete bipartite graph [15].

In this paper, we focus on the toughness of the corona of two graphs. If for each vertex $x$ in a graph $G$, we introduce a new vertex $x^{\prime}$ and join $x$ and $x^{\prime}$ by an edge, the resulting graph is called the corona of $G$. The operation of adding one vertex for each vertex of $G$ and connecting them by an edge can be generalized as follows. The corona of any two graphs $G$ and $H$, denoted by $G \circ H$, is the graph obtained by taking one copy of $G$ and $|V(G)|$ copies of $H$, and then joining the $i$ th vertex of $G$ to every vertex in the $i$ th copy of $H$. Observe that the particular case in which $H=K_{1}$, the graph $G \circ K_{1}$ is called the corona of $G$. This notion was introduced in [14].

There exist several kinds of interconnection networks whose structure can be modelled in terms of the corona of two predetermined networks. This is the case, for instance, of electric networks distributed in a big city where each transformer must guarantee the energy supply of its catchment area. In order to optimize resources, the distribution of transformers is made by dividing the city in catchment areas of the same entity. Thus, in terms of graph theory, the structure to be analysed consists of a network transformers, modelled by a graph $G$, where each transformer is connected with its catchment area, modelled by the graph $H$. The resultant graph is the corona $G \circ H$ of $G$ and $H$. In the maintenance of electric networks, it is relevant to avoid the disruption of the energy supply, but when the failure of some nodes produces the rupture of the network, the greater the number of fragments in which the network has been divided, the greater the cost of reconstruction.

The relationship between the cardinality of a cutset of a graph $G$ and the remaining components after disruption is analysed by the notion of toughness, defined above. So our aim in this work is to determine the toughness of the corona $G \circ H$ of two connected graphs $G$ and $H$ in terms of their known parameters. As a consequence, we will deduce the exact value of the corona of some families of graphs involving paths, cycles, stars, wheels or complete graphs.

\section{Notations and remarks}

Let $G$ and $H$ be two connected graphs on $m$ and $n$ vertices, respectively. Let us set $V(G)=$ $\left\{v_{1}, \ldots, v_{m}\right\}$ and denote by $H_{i}$ the copy of $H$ that is joined to vertex $v_{i}$ of $G$ in $G \circ H$. Thus, every cutset $S$ of $G \circ H$ will henceforth be expressed as $S=S_{0} \cup \bigcup_{i=1}^{m} S_{i}$, where $S_{0} \subseteq V(G)$ and $S_{i} \subseteq V\left(H_{i}\right)$, for $i=1, \ldots, m$. We denote by $\omega_{0}=\omega\left(G-S_{0}\right), \omega_{i}=\omega\left(H_{i}-S_{i}\right), i=1, \ldots, m$, that is, the number of component of $G-S_{0}$ and $H_{i}-S_{i}, i=1, \ldots, m$, respectively.

A cutset of $G \circ H$ such that $|S| / \omega(G \circ H-S)=\tau(G \circ H)$ will be called a $\tau$-cut of $G \circ H$. Let us see some remarks on the $\tau$-cut of the corona of two graphs. 
Remark 2.1 If $S=S_{0} \cup \bigcup_{i=1}^{m} S_{i}$ is a cutset of the corona $G \circ H$ of two connected graphs $G$ and
$H$, then $S_{0} \neq \emptyset$.

Proof If $S_{0}=\emptyset$, then every vertex of $G \circ H-S$ either is in $V(G)$ or is adjacent to one vertex of $G$; hence, $G \circ H-S$ is connected, against the fact that $S$ is a cutset of $G \circ H$.

Remark 2.2 Let $S=S_{0} \cup \bigcup_{i=1}^{m} S_{i}$ be a $\tau$-cut of the corona $G \circ H$ of two connected graphs $G$, $H$. If $v_{j} \in S_{0}$, then either $S_{j}=\emptyset$ or $S_{j}$ is a cutset of $H_{j}$.

Proof Let $v_{j} \in S_{0}$ and suppose by contradiction that $S_{j} \neq \emptyset$ is not a cutset of $H_{j}$. Let us consider the set $S^{*}=S \backslash S_{j}$. Observe that either $H_{j}-S_{j}$ is a component of $G \circ H-S$ or $S_{j}=V\left(H_{j}\right)$ and $H_{j}$ is a component of $G \circ H-S^{*}$. Thus, $\omega\left(G \circ H-S^{*}\right) \geq \omega(G \circ H-S)$, and therefore,

$$
\frac{\left|S^{*}\right|}{\omega\left(G \circ H-S^{*}\right)} \leq \frac{|S|-\left|S_{j}\right|}{\omega(G \circ H-S)}<\frac{|S|}{\omega(G \circ H-S)}=\tau(G \circ H-S),
$$

which contradicts the hypothesis that $S$ is a $\tau$-cut of $G \circ H$. Then either $S_{j}=\emptyset$ or $S_{j}$ is a cutset of $H_{j}$.

Remark 2.3 Let $S=S_{0} \cup \bigcup_{i=1}^{m} S_{i}$ be a $\tau$-cut of the corona $G \circ H$ of two connected graphs $G$, $H$. If $v_{j} \notin S_{0}$, then $S_{j}=\emptyset$.

Proof Let $v_{j} \notin S_{0}$ and suppose by contradiction that $S_{j} \neq \emptyset$. Let us consider the set $S^{*}=S \backslash S_{j}$. Observe that either $H_{j}-S_{j}$ belongs to the component of $G \circ H-S$ that contains vertex $v_{j}$ or $S_{j}=V\left(H_{j}\right)$ and $H_{j}$ belongs to the component of $G \circ H-S^{*}$ that contains vertex $v_{j}$. Thus, $\omega\left(G \circ H-S^{*}\right)=\omega(G \circ H-S)$, and therefore,

$$
\frac{\left|S^{*}\right|}{\omega\left(G \circ H-S^{*}\right)}=\frac{|S|-\left|S_{j}\right|}{\omega(G \circ H-S)}<\frac{|S|}{\omega(G \circ H-S)}=\tau(G \circ H-S),
$$

which is again a contradiction with the fact that $S$ is a $\tau$-cut of $G \circ H$. Then $S_{j}=\emptyset$.

Let $S=S_{0} \cup \bigcup_{i=1}^{m} S_{i}$ be a $\tau$-cut of $G \circ H$. From now on, we may assume without loss of generality that the vertices of the set $V(G)=\left\{v_{1}, \ldots, v_{m}\right\}$ are ordered so that $\left|S_{1}\right| \geq \cdots \geq\left|S_{m}\right|$. Let $k \in\{1, \ldots, m\}$ be the maximum integer such that $S_{i} \neq \emptyset$ for all $i=1, \ldots, k$. Then, as an immediate consequence of Remarks 2.1-2.3, it follows that $|S|=\left|S_{0}\right|+\sum_{i=1}^{k}\left|S_{i}\right|$ and $\omega(G \circ$ $H-S)=\omega_{0}+\sum_{i=1}^{k} \omega_{i}+\left|S_{0}\right|-k$.

\section{Main results}

Let $G, H$ be two connected graphs on $m$ and $n$ vertices, respectively. Our purpose is to determine the toughness of the corona $G \circ H$ of $G$ and $H$. To begin with, given a $\tau$-cut of $G \circ H$, the first question that we must answer is whether every copy of graph $H$ can be disconnected in the same way. The following lemma provides an answer to this question.

Lemma 3.1 Let $G$ and $H$ be two connected graphs of order $m$ and $n$, respectively, and let $S=$ $S_{0} \cup \bigcup_{i=1}^{m} S_{i}$ be a $\tau$-cut of $G \circ H$ of minimum cardinality. If $S_{i} \neq \emptyset, S_{j} \neq \emptyset$, for $i, j=1, \ldots, m$ with $i \neq j$, then $\left|S_{i}\right|=\left|S_{j}\right|$ and $\omega_{i}=\omega_{j}$. 
Proof Let us consider the vertex set $V(G)=\left\{v_{1}, \ldots, v_{m}\right\}$ ordered so that $\left|S_{1}\right| \geq \cdots \geq\left|S_{m}\right|$, and let $k \in\{1, \ldots, m\}$ be the maximum integer such that $S_{i} \neq \emptyset$ for all $i=1, \ldots, k$. Thus, $|S|=\left|S_{0}\right|+\sum_{i=1}^{k}\left|S_{i}\right|$. Since $S$ is a $\tau$-cut of $G \circ H$, we have

$$
\tau(G \circ H)=\frac{\left|S_{0}\right|+\sum_{i=1}^{k}\left|S_{i}\right|}{\omega_{0}+\sum_{i=1}^{k} \omega_{i}+\left|S_{0}\right|-k} \leq \frac{\left|S_{0}\right|+k\left|S_{\ell}\right|}{\omega_{0}+k \omega_{\ell}+\left|S_{0}\right|-k}, \quad \text { for every } \ell=1, \ldots, k,
$$

yielding that

$$
\begin{aligned}
& \left(\left|S_{0}\right|+\sum_{i=1}^{k}\left|S_{i}\right|\right) k \omega_{\ell}+\left(\omega_{0}+\left|S_{0}\right|-k\right) \sum_{i=1}^{k}\left|S_{i}\right| \\
& \quad \leq\left|S_{0}\right| \sum_{i=1}^{k} \omega_{i}+\left(\omega_{0}+\sum_{i=1}^{k} \omega_{i}+\left|S_{0}\right|-k\right) k\left|S_{\ell}\right|, \quad \text { for } \ell=1, \ldots, k .
\end{aligned}
$$

By taking summation in Equation (2), we deduce that

$$
\begin{aligned}
& \left(\left|S_{0}\right|+\sum_{i=1}^{k}\left|S_{i}\right|\right) k \sum_{\ell=1}^{k} \omega_{\ell}+k\left(\omega_{0}+\left|S_{0}\right|-k\right) \sum_{i=1}^{k}\left|S_{i}\right| \\
& \leq k\left|S_{0}\right| \sum_{i=1}^{k} \omega_{i}+\left(\omega_{0}+\sum_{i=1}^{k} \omega_{i}+\left|S_{0}\right|-k\right) k \sum_{\ell=1}^{k}\left|S_{\ell}\right| \\
& =\left(\left|S_{0}\right|+\sum_{\ell=1}^{k}\left|S_{\ell}\right|\right) k \sum_{i=1}^{k} \omega_{i}+k\left(\omega_{0}+\left|S_{0}\right|-k\right) \sum_{\ell=1}^{k}\left|S_{\ell}\right|,
\end{aligned}
$$

which implies that all the inequalities of Equation (2) become equalities, and therefore, all the inequalities of Equation (1) become equalities. Thus,

$$
\tau(G \circ H)=\frac{\left|S_{0}\right|+k\left|S_{i}\right|}{\omega_{0}+k \omega_{i}+\left|S_{0}\right|-k}=\frac{\left|S_{0}\right|+k\left|S_{j}\right|}{\omega_{0}+k \omega_{j}+\left|S_{0}\right|-k}, \quad \text { for all } i, j=1, \ldots, k,
$$

which means that the set $S^{*}=S_{0} \cup \bigcup_{i=1}^{k} S_{i}^{*}$, where $S_{i}^{*}=S_{k}$, for all $i=1, \ldots, k$, is also a $\tau$-cut. Hence,

$$
|S|=\left|S_{0}\right|+\sum_{i=1}^{k}\left|S_{i}\right| \geq\left|S_{0}\right|+k\left|S_{k}\right|=\left|S^{*}\right|,
$$

leading to $\left|S_{1}\right|=\cdots=\left|S_{k}\right|$ because $S$ has minimum cardinality. Moreover, given any two subsets $S_{i}, S_{j}$, with $i, j \in\{1, \ldots, k\}$ and $i \neq j$, from Equation (3), it is clear that $\omega_{i}=\omega_{j}$. Then the result holds.

Given a $\tau$-cut $S=S_{0} \cup \bigcup_{i=1}^{m} S_{i}$ of $G \circ H$ with minimum cardinality, by Lemma 3.1, we may assume without loss of generality that for each $i=1, \ldots, m$, either $S_{i}=\emptyset$ or $S_{i}=S_{H}$, for some $S_{H} \subset V(H)$. Furthermore, it follows that either $\omega\left(H_{i}-S_{i}\right)=1$ (if $S_{i}=\emptyset$ ) or $\omega\left(H_{i}-S_{i}\right)=$ $\omega\left(H-S_{H}\right)$ (if $\left.S_{i}=S_{H}\right)$. 
To upper bound the index of toughness of $G \circ H$, it is enough to find a cutset $S$ of $G \circ H$ and compute $|S| / \omega(G \circ H-S)$. There are some alternatives in the choice of such a cutset, as the following proposition shows.

Proposition 3.1 Let $G$ and $H$ be two connected graphs of order $m$ and $n$, respectively. Let $S_{H} \subset V(H)$ be any cutset of $H$ of cardinality $\left|S_{H}\right|=p$ and denote by $q=\omega\left(H-S_{H}\right)$. Then

$$
\tau(G \circ H) \leq \min \left\{\frac{1}{2}, \frac{\tau(G)}{1+\tau(G)}, \frac{1+p}{1+q}, \frac{1+p}{1 / \tau(G)+q}\right\} .
$$

Proof First, let $v_{j}$ be any vertex of $V(G)$ and let us consider the set $S=\left\{v_{j}\right\}$ in $G \circ H$. Then $S$ is a cutset of $G \circ H$ since $v_{j}$ separates the copy $H_{j}$ of $H$ from $G \circ H-\left(\left\{v_{j}\right\} \cup V\left(H_{j}\right)\right)$. Furthermore, $G \circ H-S$ has at least two components, i.e. $\omega(G \circ H-S)=1+\omega(G \circ H-$ $\left.\left(\left\{v_{j}\right\} \cup V\left(H_{j}\right)\right)\right) \geq 2$, yielding that $\tau(G \circ H) \leq|S| / \omega(G \circ H-S) \leq 1 / 2$.

Second, let $S \subset V(G)$ be a $\tau$-cut of $G$. Then $S$ is a cutset of $G \circ H$ and $\omega(G \circ H-S)=$ $\omega(G-S)+|S|$, and therefore,

$$
\tau(G \circ H) \leq \frac{|S|}{\omega(G \circ H-S)}=\frac{|S|}{\omega(G-S)+|S|}=\frac{|S| / \omega(G-S)}{1+|S| / \omega(G-S)}=\frac{\tau(G)}{1+\tau(G)}
$$

Third, let $S_{H} \subset V(H)$ be any cutset of $H$ of cardinality $\left|S_{H}\right|=p$ and denote by $q=\omega(H-$ $\left.S_{H}\right)$. Take any vertex $v_{j} \in V(G)$ and set $S_{j}=S_{H} \subset V\left(H_{j}\right)$. Let us consider the vertex set $S=$ $\left\{v_{j}\right\} \cup S_{j}$ and observe that $S$ is a cutset of $G \circ H$. Indeed, $\omega(G \circ H-S)=\omega\left(G-v_{j}\right)+\omega\left(H_{j}-\right.$ $\left.S_{j}\right) \geq 1+\omega\left(H_{j}-S_{j}\right)$. Thus, it follows that

$$
\tau(G \circ H) \leq \frac{|S|}{\omega(G \circ H-S)} \leq \frac{1+\left|S_{j}\right|}{1+\omega\left(H_{j}-S_{j}\right)}=\frac{1+p}{1+q} .
$$

Finally, take any cutset $S_{H} \subset V(H)$ of $H$ of cardinality $\left|S_{H}\right|=p$ and denote by $q=\omega\left(H-S_{H}\right)$. Let $S_{0}=\left\{u_{1}, \ldots, u_{\left|S_{0}\right|}\right\} \subset V(G)$ be a $\tau$-cut of $G$ and denote by $H_{i}$ the copy of $H$ joined to vertex $u_{i}$ in $G \circ H$, for $i=1, \ldots,\left|S_{0}\right|$. Let us consider the vertex set $S=S_{0} \cup \bigcup_{i=1}^{\left|S_{0}\right|} S_{i}$, where $S_{i}=S_{H}$, for every $i=1, \ldots,\left|S_{0}\right|$. Clearly $S$ is a cutset of $G \circ H$ and $\omega(G \circ H-S)=\omega(G-$ $\left.S_{0}\right)+\left|S_{0}\right| \omega\left(H-S_{H}\right)$. Hence,

$$
\begin{aligned}
\tau(G \circ H) \leq \frac{|S|}{\omega(G \circ H-S)} & =\frac{\left|S_{0}\right|+\left|S_{0}\right|\left|S_{H}\right|}{\omega\left(G-S_{0}\right)+\left|S_{0}\right| \omega\left(H-S_{H}\right)} \\
& =\frac{\left|S_{0}\right|(1+p)}{\omega\left(G-S_{0}\right)+\left|S_{0}\right| q} \\
& =\frac{\tau(G)(1+p)}{1+\tau(G) q} \\
& =\frac{1+p}{1 / \tau(G)+q} .
\end{aligned}
$$

Thus, $\tau(G \circ H) \leq \min \{1 / 2, \tau(G) / 1+\tau(G),(1+p) /(1+q),(1+p) /(1 / \tau(G)+q)\}$ and the result holds.

The next result gives a necessary condition for a $\tau$-cut of $G \circ H$ to contain vertices of some copy $H_{i}$. 
Lemma 3.2 Let $G$ and $H$ be two connected graphs of order $m$ and $n$, respectively, and let $S=S_{0} \cup \bigcup_{i=1}^{m} S_{i}$ be a $\tau$-cut of $G \circ H$ of minimum cardinality. If $S_{j} \neq \emptyset$ for some $j=1, \ldots, m$, then $\left|S_{j}\right| / \omega\left(H_{j}-S_{j}\right)<1 / 2$.

Proof From Lemma 3.1, there exists a vertex set $S_{H} \subset V(H)$ such that either $S_{i}=\emptyset$ or $S_{i}=S_{H}$, for every $i=1, \ldots, m$. So without loss of generality we may assume that there is an integer $k \in$ $\{1, \ldots, m\}$ such that $S=S_{0} \cup \bigcup_{i=1}^{k} S_{H}$; that is, $S_{i}=S_{H}$ if $i \in\{1, \ldots, k\}$ and $S_{i}=\emptyset$ otherwise. Therefore, it is enough to us to prove that $\left|S_{H}\right| / \omega\left(H-S_{H}\right)<1 / 2$. To clarify expressions, denote by $\omega_{0}=\omega\left(G-S_{0}\right)$ and $\omega_{H}=\omega\left(H-S_{H}\right)$. By applying Remark 2.1 , we know that $S_{0} \neq \emptyset$, and from Remarks 2.2 and 2.3, it follows that $k \leq\left|S_{0}\right|$. Thus, $|S|=\left|S_{0}\right|+k\left|S_{H}\right|$ and $\omega(G \circ H-S)=$ $\omega_{0}+k \omega_{H}+\left|S_{0}\right|-k$. By applying Proposition 3.1, we know that $\tau(G \circ H) \leq 1 / 2$, which implies that

$$
\frac{|S|}{\omega(G \circ H-S)}=\frac{\left|S_{0}\right|+k\left|S_{H}\right|}{\omega_{0}+k \omega_{H}+\left|S_{0}\right|-k} \leq \frac{1}{2},
$$

leading to

$$
\frac{\left|S_{H}\right|}{\omega_{H}} \leq \frac{1}{2}+\frac{\omega_{0}-\left(\left|S_{0}\right|+k\right)}{2 k \omega_{H}} .
$$

Since $S_{0} \neq \emptyset$ by Remark 2.1 and $k \geq 1$, if $S_{0}$ is not a cutset of $G$ then $\omega_{0} \leq 1$ (i.e. $\omega_{0}=0$ if $S_{0}=$ $V(G)$, and $\omega_{0}=1$ otherwise). Hence, applying inequality $\omega_{0}-\left(\left|S_{0}\right|+k\right)<0$ in Equation (4), we have $\left|S_{H}\right| / \omega_{H}<1 / 2$. Thus, suppose that $S_{0} \subset V(G)$ is a cutset of $G$.

First assume that $\left|S_{0}\right| / \omega_{0} \geq 1$. This means that $\omega_{0}-\left(\left|S_{0}\right|+k\right)<\omega_{0}-\left|S_{0}\right| \leq 0$, yielding in Equation (4) that $\left|S_{H}\right| / \omega_{H}<1 / 2$.

Second assume that $\left|S_{0}\right| / \omega_{0}<1$. Since $S_{0}$ is a cutset of $G$, then it is also a cutset of $G \circ H$ and $\omega\left(G \circ H-S_{0}\right)=\omega_{0}+\left|S_{0}\right|$. Therefore, by using that $S$ is a $\tau$-cut of $G \circ H$, it follows that

$$
\frac{\left|S_{0}\right|}{\omega_{0}+\left|S_{0}\right|} \geq \tau(G \circ H)=\frac{\left|S_{0}\right|+k\left|S_{H}\right|}{\omega_{0}+k \omega_{H}+\left|S_{0}\right|-k}>\frac{\left|S_{0}\right|+k\left|S_{H}\right|}{\omega_{0}+k \omega_{H}+\left|S_{0}\right|} .
$$

Combining the first and the last members of Equation (5), we deduce that

$$
\frac{\left|S_{H}\right|}{\omega_{H}}<\frac{\left|S_{0}\right|}{\omega_{0}+\left|S_{0}\right|}=\frac{\left|S_{0}\right| / \omega_{0}}{1+\left|S_{0}\right| / \omega_{0}}<\frac{1}{2}
$$

because $\left|S_{0}\right| / \omega_{0}<1$. This concludes the proof.

From these previous results, the next theorem follows where the toughness of the corona $G \circ H$ of two connected graphs is determined in terms of some parameters of $G$ and $H$.

THEOREM 3.1 Let $G$ and $H$ be two connected graphs of order $m$ and $n$, respectively. Then the following assertions hold:

(i) If $\tau(G) \geq 1$ and $\tau(H) \geq 1 / 2$, then $\tau(G \circ H)=1 / 2$.

(ii) If $\tau(G)<1$ and $\tau(H) \geq 1 / 2$, then $\tau(G \circ H)=\tau(G) /(1+\tau(G))$.

(iii) If $\tau(G) \geq 1$ and $\tau(H)<1 / 2$, then $\tau(G \circ H)=\min _{S_{H} \in J(H)}\left\{\frac{1+\left|S_{H}\right|}{1+\omega\left(H-S_{H}\right)}\right\}$.

(iv) If $\tau(G)<1$ and $\tau(H)<1 / 2$, then

$$
\tau(G \circ H)=\min \left\{\frac{\tau(G)}{1+\tau(G)}, \min _{S_{H} \in J(H)} \frac{1+\left|S_{H}\right|}{\frac{1}{\tau(G)}+\omega\left(H-S_{H}\right)}\right\} .
$$

Proof Let $S=S_{0} \cup \bigcup_{i=1}^{m} S_{i}$ be a $\tau$-cut of $G \circ H$. Without loss of generality, we may assume that $V(G)=\left\{v_{1}, \ldots, v_{m}\right\}$, where the vertices are numbered so that $\left|S_{i}\right| \geq\left|S_{i+1}\right|$, for all $i=$ $1, \ldots, m-1$. We may also suppose that $S$ has minimum cardinality over all the $\tau$-cuts of $G \circ H$. 
First, assume that $\tau(H) \geq 1 / 2$. Then by applying Lemma 3.2, we deduce that $S_{i}=\emptyset$, for all $i=1, \ldots, m$, hence, $S=S_{0}$. This implies that $\tau(G \circ H)=|S| / \omega(G \circ H-S)=\left|S_{0}\right| / \omega_{0}+$ $\left|S_{0}\right|$. Note that $S_{0} \neq V(G)$, because otherwise, $\omega_{0}=0$; that is, $\omega\left(G \circ H-S_{0}\right)=\left|S_{0}\right|$, and therefore, $\tau(G \circ H)=1$, which is a contradiction to Proposition 3.1. Thus, $S_{0} \subset V(G)$, which means that $\omega_{0} \geq 1$, yielding $\omega\left(G \circ H-S_{0}\right) \geq 1+\left|S_{0}\right|$. If $S_{0}$ is not a cutset of $G$, then $\omega_{0}=1$, and therefore, $\tau(G \circ H)=\left|S_{0}\right| / \omega_{0}+\left|S_{0}\right|=\left|S_{0}\right| / 1+\left|S_{0}\right| \geq 1 / 2$. If $S_{0}$ is a cutset of $G$ then $\omega_{0} \geq 2$, and therefore,

$$
\tau(G \circ H)=\frac{\left|S_{0}\right|}{\omega_{0}+\left|S_{0}\right|}=\frac{\left|S_{0}\right| / \omega_{0}}{1+\left|S_{0}\right| / \omega_{0}} \geq \frac{\tau(G)}{1+\tau(G)} .
$$

Hence, $\tau(G \circ H) \geq \min \{1 / 2, \tau(G) /(1+\tau(G))\}$. Moreover, by Proposition 3.1, we have $\tau(G \circ$ $H) \leq \min \{1 / 2, \tau(G) /(1+\tau(G))\}$, leading to

$$
\tau(G \circ H)=\min \left\{\frac{1}{2}, \frac{\tau(G)}{1+\tau(G)}\right\}= \begin{cases}\frac{1}{2}, & \text { if } \tau(G) \geq 1 \\ \frac{\tau(G)}{1+\tau(G)}, & \text { if } \tau(G)<1\end{cases}
$$

which proves items (i) and (ii).

Second, assume that $\tau(H)<1 / 2$. If $S_{1}=\emptyset$, then $S_{i}=\emptyset$ for every $i=1, \ldots, m$, and reasoning as above, we prove that

$$
\tau(G \circ H) \geq \min \left\{\frac{1}{2}, \frac{\tau(G)}{1+\tau(G)}\right\}= \begin{cases}\frac{1}{2}, & \text { if } \tau(G) \geq 1 \\ \frac{\tau(G)}{1+\tau(G)}, & \text { if } \tau(G)<1 .\end{cases}
$$

Thus, suppose that $S_{1} \neq \emptyset$, then by Lemma 3.1, we may assume that there exists an integer $k \in\{1, \ldots, m\}$ and a non-empty vertex set $S_{H} \subset V(H)$ such that $S_{i}=S_{H}$ if $i \leq k$, and $S_{i}=\emptyset$ otherwise. Further, from Lemma 3.2, it follows that $\left|S_{H}\right| / \omega\left(H-S_{H}\right)<1 / 2$. Again to clarify expressions, denote by $\omega_{H}=\omega\left(H-S_{H}\right)$. Notice that $k \leq\left|S_{0}\right|$ by Remarks 2.2 and 2.3, and therefore, $\tau(G \circ H)=\left(\left|S_{0}\right|+k\left|S_{H}\right|\right) /\left(\omega_{0}+k \omega_{H}+\left|S_{0}\right|-k\right)$. Since $S_{0}$ is also a cutset of $G \circ H$ and $S$ is a $\tau$-cut of $G \circ H$ of minimum cardinality, we have

$$
\frac{\left|S_{0}\right|}{\omega_{0}+\left|S_{0}\right|}>\tau(G \circ H)=\frac{\left|S_{0}\right|+k\left|S_{H}\right|}{\omega_{0}+k \omega_{H}+\left|S_{0}\right|-k},
$$

yielding that

$$
\left|S_{H}\right|\left(\omega_{0}+\left|S_{0}\right|\right)-\left|S_{0}\right|\left(\omega_{H}-1\right)<0 .
$$

The function $f(k)=\left(\left|S_{0}\right|+k\left|S_{H}\right|\right) /\left(\omega_{0}+k \omega_{H}+\left|S_{0}\right|-k\right)$ has a derivate

$$
\frac{\mathrm{d} f}{\mathrm{~d} k}=\frac{\left|S_{H}\right|\left(\omega_{0}+\left|S_{0}\right|\right)-\left|S_{0}\right|\left(\omega_{H}-1\right)}{\left(\omega_{0}+k \omega_{H}+\left|S_{0}\right|-k\right)^{2}},
$$

and by Equation (7), we deduce that $f(k)$ is decreasing in $k$. Hence,

$$
\tau(G \circ H)=f(k) \geq f\left(\left|S_{0}\right|\right)=\frac{\left|S_{0}\right|\left(1+\left|S_{H}\right|\right)}{\omega_{0}+\left|S_{0}\right| \omega_{H}} .
$$


If $S_{0}$ is not a cutset of $G$ then $\omega_{0} \leq 1\left(\omega_{0}=0\right.$ if $S_{0}=V(G)$, and $\omega_{0}=1$ otherwise), and from Equation (8), it follows that

$$
\begin{aligned}
\tau(G \circ H) \geq \frac{\left|S_{0}\right|\left(1+\left|S_{H}\right|\right)}{1+\left|S_{0}\right| \omega_{H}} & =\frac{1+\left|S_{H}\right|}{1 /\left|S_{0}\right|+\omega_{H}} \\
& \geq \frac{1+\left|S_{H}\right|}{1+\omega_{H}} \\
& \geq \min _{S_{H} \in J(H)}\left\{\frac{1+\left|S_{H}\right|}{1+\omega_{H}}\right\} .
\end{aligned}
$$

If $S_{0}$ is a cutset of $G$ then $\left|S_{0}\right| / \omega_{0} \geq \tau(G)$, and therefore, from Equation (8), it follows that

$$
\begin{aligned}
\tau(G \circ H) \geq \frac{\left|S_{0}\right|\left(1+\left|S_{H}\right|\right)}{\omega_{0}+\left|S_{0}\right| \omega_{H}} & =\frac{1+\left|S_{H}\right|}{\omega_{0} /\left|S_{0}\right|+\omega_{H}} \\
& \geq \frac{1+\left|S_{H}\right|}{1 / \tau(G)+\omega_{H}} \\
& \geq \min _{S_{H} \in J(H)}\left\{\frac{1+\left|S_{H}\right|}{1 / \tau(G)+\omega_{H}}\right\} .
\end{aligned}
$$

(iii) Suppose that $\tau(G) \geq 1$. Then combining Equations (6), (9) and (10), we deduce that

$$
\begin{aligned}
\tau(G \circ H) & \geq \min \left\{\frac{1}{2}, \min _{S_{H} \in J(H)}\left\{\frac{1+\left|S_{H}\right|}{1+\omega_{H}}\right\}, \min _{S_{H} \in J(H)}\left\{\frac{1+\left|S_{H}\right|}{1 / \tau(G)+\omega_{H}}\right\}\right\} \\
& =\min \left\{\frac{1}{2}, \min _{S_{H} \in J(H)}\left\{\frac{1+\left|S_{H}\right|}{1+\omega_{H}}\right\}\right\} .
\end{aligned}
$$

Since $\tau(H)<1 / 2$, there exists a cutset $S_{H} \subset V(H)$ such that $\left|S_{H}\right| / \omega_{H}<1 / 2$, which implies that $2\left|S_{H}\right|+1 \leq \omega_{H}$. Then

$$
\frac{1+\left|S_{H}\right|}{1+\omega_{H}} \leq \frac{\omega_{H}-\left|S_{H}\right|}{1+\omega_{H}}=\frac{1+\omega_{H}-\left(1+\left|S_{H}\right|\right)}{1+\omega_{H}}=1-\frac{1+\left|S_{H}\right|}{1+\omega_{H}},
$$

which means that $\left(1+\left|S_{H}\right|\right) /\left(1+\omega_{H}\right) \leq 1 / 2$ and therefore,

$$
\tau(G \circ H)=\min _{S_{H} \in J(H)}\left\{\frac{1+\left|S_{H}\right|}{1+\omega_{H}}\right\} .
$$

(iv) Now suppose that $\tau(G)<1$, then from Equations (6), (9) and (10), it follows that

$$
\tau(G \circ H) \geq \min \left\{\frac{\tau(G)}{1+\tau(G)}, \min _{S_{H} \in J(H)}\left\{\frac{1+\left|S_{H}\right|}{1 / \tau(G)+\omega_{H}}\right\}\right\} .
$$

As a consequence of Theorem 3.1, the toughness of the corona of some families of graphs can be derived. Let $n \geq 3$ be an integer. Let us denote by $P_{n}$ and $C_{n}$ the path and the cycle with $n$ vertices, respectively, by $S_{n}$ the complete bipartite graph $K_{1, n-1}$, by $W_{1, n}$ the wheel with $n+1$ vertices and by $K_{n}$ the complete graph of order $n$. As a consequence of Theorem 3.1, the toughness of the corona of two graphs, one of them being a complete graph, is deduced. Further, in Table 1, we can find the toughness of the corona of two graphs belonging to some of these families: stars, paths, cycles, wheels and complete graphs. 
Table 1. The toughness of the corona of some families of graphs.

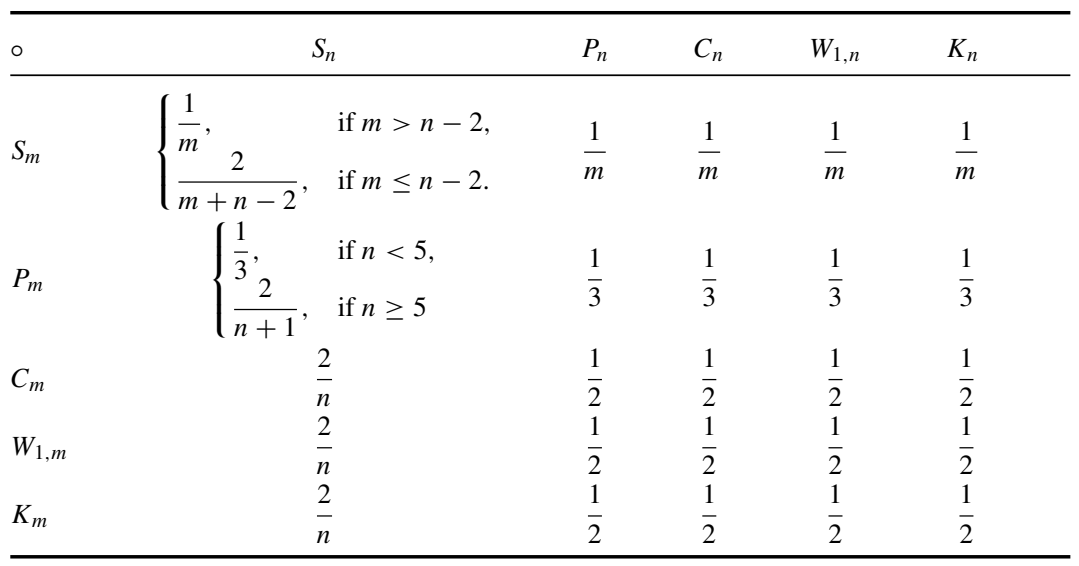

Corollary 3.1 Let $m \geq 3, n \geq 3$ be two integers and let $G$ and $H$ be two connected graphs. Then the following assertions hold:

(i) $\tau\left(G \circ K_{n}\right)= \begin{cases}\frac{1}{2}, & \text { if } \tau(G) \geq 1, \\ \frac{\tau(G)}{1+\tau(G)}, & \text { if } \tau(G)<1 .\end{cases}$
(ii) $\tau\left(K_{m} \circ H\right)= \begin{cases}\frac{1}{2}, & \text { if } \tau(H) \geq 1 / 2, \\ \min _{S_{H} \subset J(H)}\left\{\frac{1+\left|S_{H}\right|}{1+\omega\left(H-S_{H}\right)}\right\}, & \text { if } \tau(H)<1 / 2 .\end{cases}$

\section{Conclusions}

To measure the vulnerability of networks more properly, there exist some parameters that focus not only the difficulty of breaking down the network but also the effect of the damage. In this work, we have studied the toughness of the corona of two graphs. This family models the structure of interconnection networks in several fields, where the reliability is one of most important features. We have determined the value of the toughness of this kind of graphs.

\section{Acknowledgement}

We want to deeply thank the anonymous referees who helped us to improve this paper. This research was supported by the Ministry of Education and Science, Spain, and the European Regional Development Fund (ERDF) under project MTM2008-06620-C03-02/MTM, Catalonian Government 1298 SGR2009 and by the Andalusian Government under project P06-FQM-01649.

\section{References}

[1] V. Aytaç, Vulnerability in graphs: The neighbour-integrity of line graphs, Int. J. Comput. Math. 82(1) (2005), pp. $35-40$.

[2] C.A. Barefoot, R. Entringer, and H. Swart, Vulnerability in graphs - a comparative survey, J. Combin. Math. Combin. Comput. 1 (1987), pp. 13-22. 
[3] D. Bauer, H. Broersma, and E. Schmeichel, Toughness in graphs - a survey, Graphs Combin. 22(1) (2006), pp. 1-35.

[4] D. Bauer, S.L. Hakimi, and E. Schmeichel, Recognizing tough graphs is N P-hard, Discrete Appl. Math. 28 (1990), pp. 191-195.

[5] F.T. Boesch, Synthesis of reliable networks - a survey, IEEE Trans. Reliab. 35 (1986), pp. 240-246.

[6] F.T. Boesch, A. Satyanarayana, and C. L. Suffel, A survey of some network reliability analysis and synthesis results, Networks 54(2) (2009), pp. 99-107.

[7] F.T. Boesch and R. Tindell, Circulants and their connectivities, J. Graph Theory 8(4) (1984), pp. 487-499.

[8] H.J. Broersma, E. Engbers, and H. Trommel, Various results on the toughness of graphs, Networks 33 (1999), pp. 233-238.

[9] G. Chartrand and L. Lesniak, Graphs and Digraphs, Chapman and Hall/CRC, USA, 2005.

[10] V. Chvátal, Tough graphs and Hamiltonian circuits, Discrete Math. 5 (1973), pp. 215-228.

[11] J. Fàbrega and M.A. Fiol, Extraconnectivity of graphs with large girth, Discrete Math 127 (1994), pp. 163-170.

[12] J. Fàbrega and M.A. Fiol, On the extraconnectivity of graphs, Discrete Math 155 (1996), pp. 49-57.

[13] R. Faudree, R. Gould, M. Jacobson, L. Lesniak, and A. Saito, Toughness, degrees and 2-factors, Discrete Math. 286 (2004), pp. 245-249.

[14] R. Frucht and F. Harary, On the corona of two graphs, Aequationes Math. 4 (1970), pp. 322-325.

[15] W.D. Goddard and H.C. Swart, On the toughness of a graph, Quaestiones Math. 13 (1990), pp. 217-232.

[16] A. Hellwig and L. Volkmann, Maximally edge-connected and vertex-connected graphs and digraphs: A survey, Discrete Math. 308(15) (2008), pp. 3265-3296.

[17] P. Katerinis and T. Wang, Toughness of graphs and 2-factors with given properties, Ars Combin. 95 (2010), pp. 161177.

[18] Y. Li, S. Zhang, and Q. Zhang, Vulnerability parameters of split graphs, Int. J. Comput. Math. 85(1) (2008), pp. $19-23$.

[19] S. Liu, On toughness and fractional ( $g, f, n)$-critical graphs, Inform. Process. Lett. 110(10) (2010), pp. 378-382.

[20] S. Liu and J. Cai, Toughness and existence offractional ( $g$, $f$ )-factors in graphs, Ars Combin. 93 (2009), pp. $305-311$.

[21] A. Mamut and E. Vumar, Vertex vulnerability parameters of Kronecker products of complete graphs, Inform. Process. Lett. 106 (2008), pp. 258-262.

[22] M. Metsidik and E. Vumar, Edge vulnerability parameters of bisplit graphs, Comput. Math. Appl. 56(7) (2008), pp. 1741-1747.

[23] M. Metsidik and E. Vumar, Toughness and matching extension in $P_{3}$-dominated graphs, Graphs Combin. 26(3) (2010), pp. 425-432.

[24] D. Moazzami and B. Salehian, Some results related to the tenacity and existence of $k$-trees, Discrete Appl. Math. 157(8) (2009), pp. 1794-1798.

[25] R.E. Pippert, On the Toughness of a Graph, Lecture Notes in Mathematics, Vol. 303, Springer, Berlin, 1972, pp. 225-233.

[26] Z. Wei and S. Zhang, Vertex-neighbour-integrity of composition graphs of paths and cycles, Int. J. Comput. Math. 85(5) (2008), pp. 727-733.

[27] S. Zhang, X. Li, and X. Han, Computing the scattering number of graphs, Int. J. Comput. Math. 79(2) (2002), pp. 179-187. 